\title{
Tumor microenvironment and radioresistance
}

\author{
Tatsuya Suwa ${ }^{1,2,3}$, Minoru Kobayashi, ${ }^{1,2}$, Jin-Min Nam ${ }^{1,2}$ and Hiroshi Harada ${ }^{1,2}$
}

\begin{abstract}
Metastasis is not the result of a random event, as cancer cells can sustain and proliferate actively only in a suitable tissue microenvironment and then form metastases. Since Dr. Stephen Paget in the United Kingdom proposed the seed and soil hypothesis of cancer metastasis based on the analogy that plant seeds germinate and grow only in appropriate soil, considerable attention has focused on both extracellular environmental factors that affect the growth of cancer cells and the tissue structure that influences the microenvironment. Malignant tumor tissues consist of not only cancer cells but also a wide variety of other cells responsible for the inflammatory response, formation of blood vessels, immune response, and support of the tumor tissue architecture, forming a complex cellular society. It is also known that the amounts of oxygen and nutrients supplied to each cell differ depending on the distance from tumor blood vessels in tumor tissue. Here, we provide an overview of the tumor microenvironment and characteristics of tumor tissues, both of which affect the malignant phenotypes and radioresistance of cancer cells, focusing on the following keywords: diversity of oxygen and nutrient microenvironment in tumor tissue, inflammation, immunity, and tumor vasculature.
\end{abstract}

\section{Heterogeneous oxygen microenvironment in malignant solid tumors and radioresistance of cancer cells \\ Tumor hypoxia}

Tumor hypoxia has attracted marked attention in radiation biology and oncology since Thomlinson and Gray reported the presence of hypoxic cells in malignant solid tumors and suggested the possibility that they exert a negative impact on the outcome of radiation therapy.

Activation of oncogenes and inactivation of tumor suppressor genes cause aberrant cell proliferation, which is a characteristic feature of cancer cells. Aberrant cell proliferation leads to an oxygen supply-demand imbalance in cancer cells and an imbalance between the rate of vascular network development and that of cancer cell proliferation in tumor tissues ${ }^{1,2}$. These are recognized as two major causative factors of the heterogeneous cellular

\footnotetext{
Correspondence: Hiroshi Harada (harada.hiroshi.5e@kyoto-u.ac.jp)

${ }^{1}$ Laboratory of Cancer Cell Biology, Graduate School of Biostudies, Kyoto

University, Yoshida Konoe-cho, Sakyo-ku, Kyoto 606-8501, Japan

2Department of Genome Repair Dynamics, Radiation Biology Center, Graduate

School of Biostudies, Kyoto University, Yoshida Konoe-cho, Sakyo-ku, Kyoto

606-8501, Japan

Full list of author information is available at the end of the article
}

oxygenation in tumor tissues (Fig. 1). It is reasonable to consider that cancer cells can obtain sufficient oxygen and nutrients only when in close proximity to tumor blood vessels (normoxic regions), because molecular oxygen can diffuse within a limited distance from functional blood vessels (Fig. 1). On the other hand, cancer cells approximately $70-100 \mu \mathrm{m}$ from tumor blood vessels can obtain the minimal level of oxygen necessary for their survival but insufficient for active proliferation (chronically hypoxic regions; Fig. 1). Yeom et al. previously suggested that hypoxic layers approximately $70-85 \mu \mathrm{m}$ from tumor blood vessels are relatively mildly hypoxic, with $\mathrm{O}_{2}$ concentrations of at least $1 \%$ but no higher than $3 \%^{3}$. On the other hand, the concentration of $\mathrm{O}_{2}$ approximately 85-100 $\mu \mathrm{m}$ from tumor blood vessels has been suggested to be $1 \%$ or less ${ }^{3}$. Cancer cells in hypoxic regions are known to produce adenosine triphosphate (ATP) mainly via oxygen-independent glycolysis rather than oxygendependent oxidative phosphorylation and generate lactate as a byproduct, leading to a unique microenvironment with a low pH. Cancer cells farther away from blood vessels inevitably die because they are deprived of oxygen and nutrients (necrotic regions; Fig. 1) ${ }^{2-4}$. A dynamic 


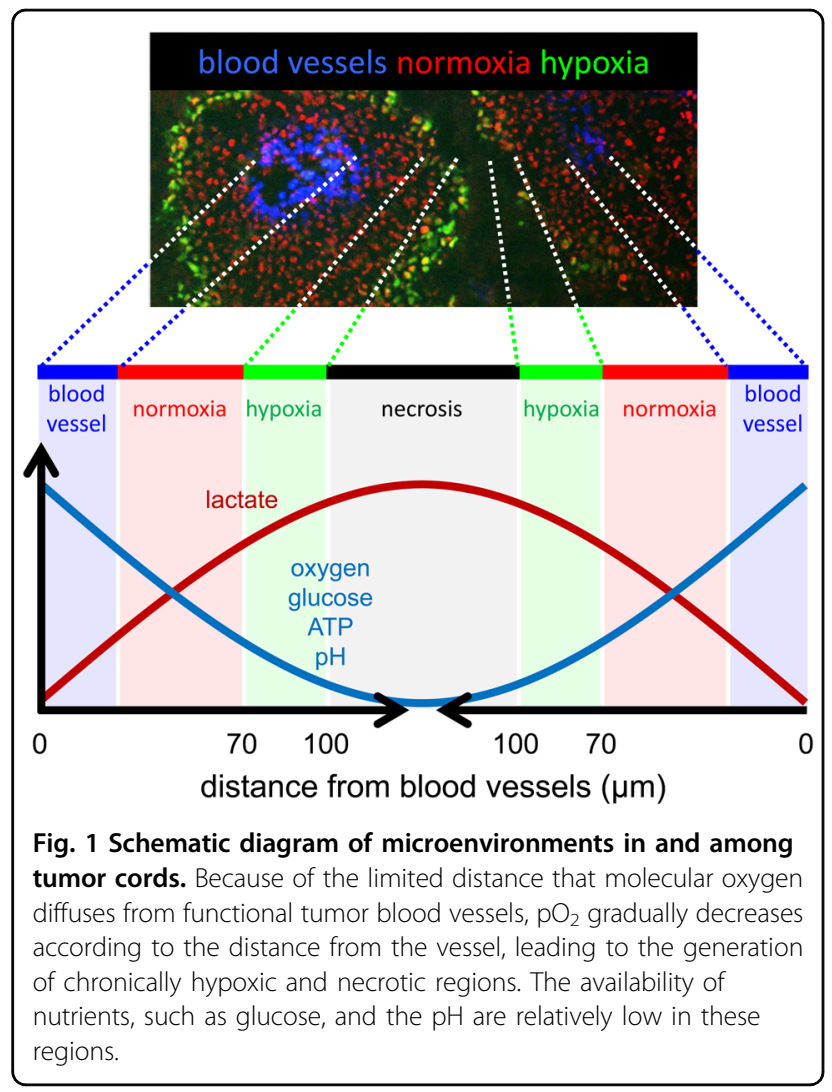

oxygen concentration gradient also exists in normal tissues, such as between the portal and central veins in liver tissue; however, a necrotic region is a unique and characteristic feature of malignant solid tumors (Fig. 1).

In addition to chronic hypoxia, another kind of hypoxia is known to exist in tumor tissues: so-called acute hypoxia. Acute hypoxia is generated-regardless of the distance from tumor blood vessels - as a result of unusual activation of proangiogenic mechanisms mediated by a hypoxia-responsive transcription factor, hypoxiainducible factor 1 (HIF-1). Cancer cells adapt to hypoxic conditions by inducing the expression of HIF-1. HIF-1 induces the expression of various proangiogenic factors, such as vascular endothelial growth factor (VEGF) and platelet-derived growth factor (PDGF). Activation of HIF1 in tumor hypoxia causes excess activation of proangiogenic signals, resulting in the development of unique vasculatures that are structurally tortuous, immature, and functionally very leaky due to poor coverage by pericytes. Interstitial pressure is inevitably high in solid tumors because of this leakiness; therefore, tumor blood vessels cannot sufficiently supply oxygen and nutrients even in well-vascularized regions. In addition, these immature tumor vessels cause transient reflux and occlusion of blood flow, also leading to transient hypoxia even within $70 \mu \mathrm{m}$ of tumor blood vessels (acutely hypoxic regions) ${ }^{2,3}$.
The concept of acute hypoxia was first recognized by Brown et al. in $1979^{5}$. Subsequent studies showed that at least $20 \%$ of cancer cells are subjected to acute hypoxia ${ }^{6-8}$.

It has been reported in clinical studies that the overall median $\mathrm{pO}_{2}$ measured by a computerized polarographic needle electrode is approximately $10 \mathrm{mmHg}$ in various malignant solid tumors, such as breast cancers and head and neck cancers 9 . In contrast, that in normal tissues has been reported to be approximately $65 \mathrm{mmHg}$. It has also been reported that the overall hypoxic fraction $\left(\mathrm{pO}_{2}<\right.$ $2.5 \mathrm{mmHg}$ ) is approximately $25 \%$ in breast cancer, while $\mathrm{pO}_{2}$ values lower than $12.5 \mathrm{mmHg}$ have never been noted in normal breast tissues ${ }^{10}$.

\section{Mechanisms underlying tumor radioresistance under hypoxia}

Through basic experiments, such as the clonogenic cell survival assay, it has been repeatedly confirmed that cultured cells are approximately $2-3$ times more radiosensitive under normoxia than under hypoxia ${ }^{11}$. Consistent with this phenomenon, known as the "oxygen effect" in the field of radiation biology, accumulated clinical evidence has also demonstrated that a large hypoxic fraction in a solid tumor is correlated with poor prognosis in cancer patients after radiation therapy ${ }^{11,12}$. For example, Kaanders et al. reported that locoregional tumor control was significantly lower for patients with head and neck cancers containing more hypoxic regions ${ }^{12}$. The cytotoxic effects of radiation are principally caused by damage to genomic DNA produced through either direct ionization of DNA or indirect action by creating water radical species that react with DNA. The oxygen effect is known to mainly result from the difference in the frequency of DNA damage produced through indirect action under hypoxia compared with normoxia.

\section{Radiochemical mechanism}

Ionizing radiation, such as $\mathrm{X}$-rays and $\gamma$-rays, produces free radicals through ionization of water. When highly reactive radicals such as hydroxyl radicals (HO•) are produced from water near genomic DNA, they cause DNA strand breaks. Molecular oxygen can stabilize reactive radicals, enabling them to cause severe DNA damage for a long period of time. In addition, molecular oxygen oxidizes the ends of damaged DNA and creates double-strand breaks that are both unrepairable and lethal $^{11,13}$. Therefore, DNA damage is less severe in the absence of oxygen ${ }^{14}$, and cancer cells in hypoxic regions exhibit radioresistance ${ }^{2}$.

\section{Radiobiological mechanism}

Biological as well as chemical mechanisms are important elements contributing to the radioresistance of hypoxic cancer cells ${ }^{15-17}$. One of the important mediators 
of these mechanisms is HIF-1. In particular, the function of HIF-1 in carbohydrate metabolic reprogramming from mitochondrial oxidative phosphorylation to accelerated glycolysis and the associated pentose phosphate pathway (PPP) is important for the radioresistance of hypoxic cancer cells ${ }^{2,18,19}$. The PPP, whose initial metabolite, glucose-6-phosphate, is supplied via glycolysis, generates pentose sugars and ribose-5-phosphate and, moreover, generates nicotinamide adenine dinucleotide phosphate (NADPH) as a byproduct ${ }^{20,21}$. Because NADPH is important for the production of an antioxidant, reduced glutathione (GSH), from glutathione-S-S-glutathione (GSSG) and because ribose-5-phosphate is important for nucleotide synthesis, HIF-1-mediated activation of the PPP is associated with the radioresistance of hypoxic cancer cells ${ }^{22}$.

We recently demonstrated the possibility that aberrant activation of upstream activators of HIF-1, such as a deubiquitinating enzyme for HIF- $1 \alpha$ protein, ubiquitin carboxyl-terminal hydrolase-L1 (UCHL1), induces the same HIF-1-dependent mechanism for radioresistance even under normoxic conditions (Fig. 2). For example, UCHL1 was found to increase the intracellular levels of GSH and NADPH even under normoxic conditions through HIF-1mediated reprogramming of carbohydrate metabolism, specifically in a PPP-dependent manner. As a result, UCHL1 induced radioresistance even under normoxic conditions through UCHL1-HIF-1-mediated metabolic reprogramming ${ }^{19,23}$. Consistent with this finding, accumulated clinical evidence has shown that overexpression of the HIF-1 protein in a solid tumor is associated with a poor prognosis in cancer patients with, e.g., head and neck, cervical, or oropharyngeal cancer after irradiation ${ }^{24-26}$.

Extensive studies have shown that radioresistance of hypoxic cancer cells is induced by the ataxia-telangiectasia mutated (ATM)-dependent DNA damage repair pathway, particularly through upregulation of homologous recombination activity ${ }^{27-32}$. In response to DNA strand breaks, the activated form of ATM phosphorylates some HRrelated factors, such as breast cancer type 1 susceptibility gene (BRCA1) and Fanconi anemia group D2 (FANCD2), triggering the DNA damage repair pathway. Because ATM expression is known to be induced also in response to hypoxic stress, it has been suggested that ATM also plays pivotal roles in the radioresistance of cancer cells under hypoxic conditions.

Another mechanism underlying the radioresistance of hypoxic cells is related to immune evasion. Because radiation therapy damages cancer cells and potentiates the production of cancer neoantigens, the therapeutic effect of radiation is greatly influenced by tumor immunity. Cancer cells express immune inhibitory molecules, such as PD-L1, and escape tumor immunity. PD-L1 expression is induced under hypoxic conditions in a HIF- 1-dependent manner and is thus thought to be one of the important mechanisms underlying the radioresistance of hypoxic cancer cells ${ }^{33}$. Thus, immune checkpoint inhibitors are expected to enhance the therapeutic effect of radiation on tumor tissues containing more hypoxic regions. Immune checkpoint inhibitors have been reported to induce vascular normalization and thus decrease the hypoxic fraction ${ }^{34,35}$; this is another reason that we expect immune checkpoint inhibitors to enhance the therapeutic effect of radiation.

\section{Cellular society within a malignant solid tumor}

An inflammatory reaction is constantly evoked in most malignant solid tumors, which causes infiltration of immune cells, angiogenesis, and proliferation of fibroblasts and produces a characteristic cellular society (Fig. 3). It has been clarified that the formation of this cellular society leads to the malignant progression and radioresistance of cancers ${ }^{36,37}$.

\section{Radioresistance of cancers mediated by tumor-associated macrophages (TAMs)}

Tumor-associated macrophages (TAMs), which exist abundantly in tumor tissues, have long been considered to exhibit antitumor effects and suppress tumor growth. However, recent studies have shown that they promote tumor growth and enhance radioresistance in various types of cancers, including breast, esophageal, cervical, and hepatic cancers ${ }^{37-39}$. Macrophages induce the expression of the inflammatory prostaglandin synthase cyclooxygenase-2 (COX-2) via the activation of nuclear factor $\kappa \mathrm{B}(\mathrm{NF}-\mathrm{kB})$, and the produced prostaglandin E2 (PGE2) has been reported to then accelerate tumor growth and induce radioresistance in cancers ${ }^{40,41}$. In addition, it has been suggested that macrophages activated by irradiation may cause radioresistance of cancer cells and facilitate tumor recurrence after radiotherapy by inducing high expression of tumor necrosis factor- $\alpha$ $(\mathrm{TNF}-\alpha)$ and promoting angiogenesis ${ }^{42}$.

The crosstalk between cancer cells and macrophages plays an important role in the growth and radioresistance of tumors. Macrophages differentiate into the M2 type when stimulated with interleukin 6 (IL6), macrophage colony stimulating factor (M-CSF), and PGE2 secreted by cancer cells. Next, proangiogenic factors, e.g., VEGF and PDGF, secreted from M2-type macrophages have been suggested to generate a microenvironment favorable for tumor growth ${ }^{43}$ and promote tumor regrowth after radiotherapy. It has also been pointed out that antitumor immunity is suppressed by the infiltration of regulatory $\mathrm{T}$ cells induced by $\mathrm{C}-\mathrm{C}$ motif chemokine ligand 22 (CCL22) secreted from M2-type macrophages ${ }^{39,44}$, which leads to increased tumor growth and cancer radioresistance ${ }^{45-48}$. 


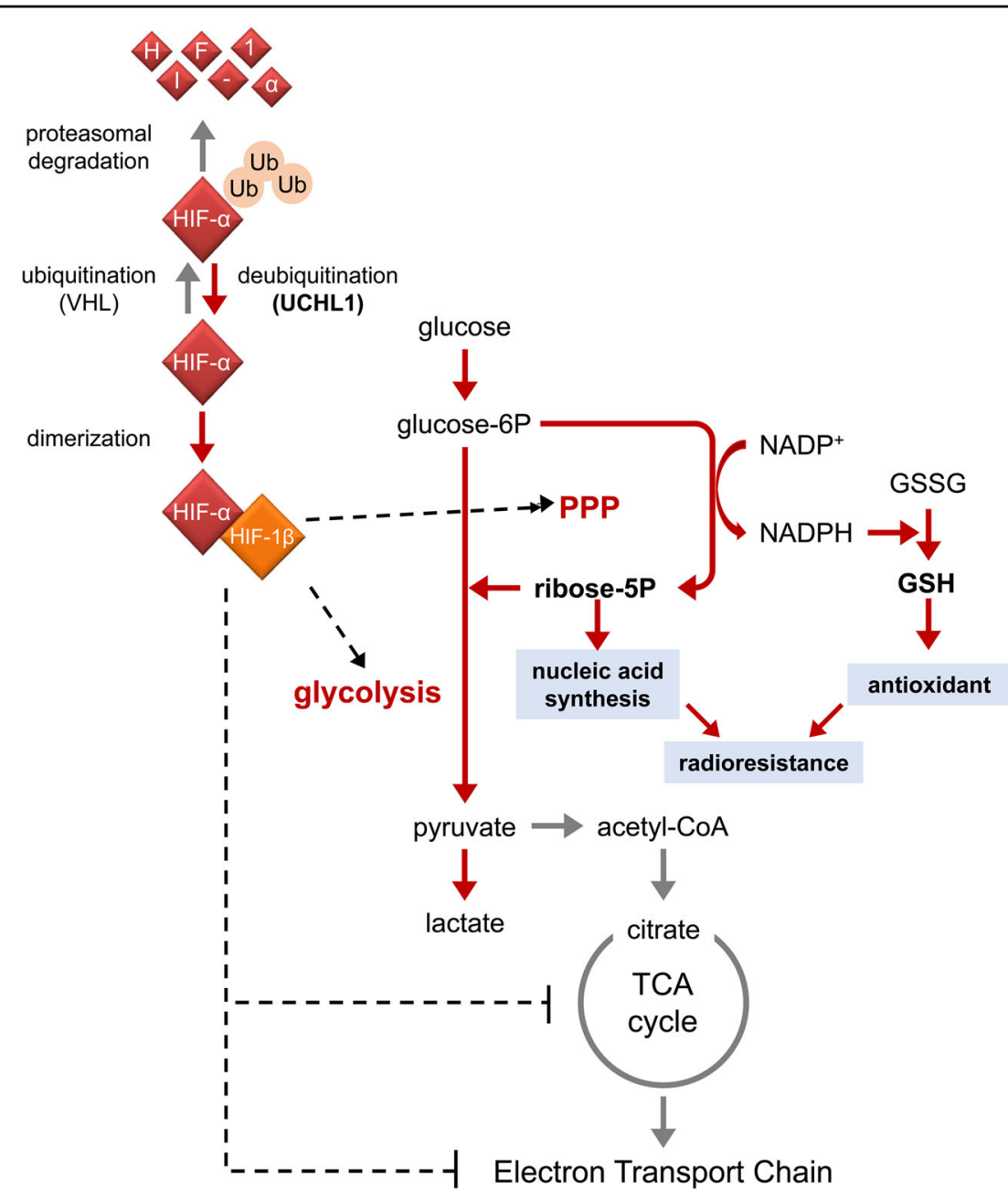

Fig. 2 Radioresistance of cells induced by UCHL1-HIF-1 pathway-dependent reprogramming of the carbohydrate metabolic pathway. HIF-1 mediates reprogramming of the glucose metabolic pathway from mitochondrial oxidative phosphorylation to glycolysis. UCHL1 causes radioresistance by inducing HIF-1-mediated reprogramming and a resulting increase in the intracellular level of GSH in a PPP-dependent manner.

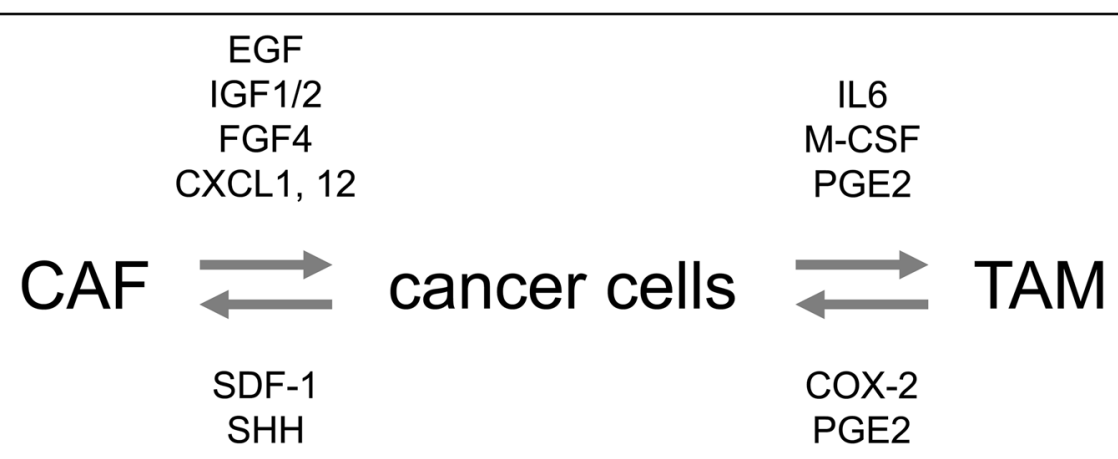

Fig. 3 Crosstalk between cancer cells and TAMs or CAFs in tumor tissue. Crosstalk between cancer cells and TAMs or CAFs is regulated by the indicated factors.

Radioresistance of cancers mediated by cancer-associated fibroblasts (CAFs)

Clinical studies on various types of cancers, such as head and neck, lung, pancreatic, and rectal cancers, have reported that the prognosis of patients after radiotherapy is poorer when tumor tissues contain more fibroblasts ${ }^{49-56}$. This clinical evidence led to two major questions: how such a fibroblast-rich microenvironment is created in tumor 
tissues, and how it enhances radioresistance. Some important findings have been reported that suggest the importance of crosstalk between cancer cells and fibroblasts in tumor tissues.

PDGF and transforming growth factor- $\beta$ (TGF- $\beta$ ), which are secreted from cancer cells, are known to induce transdifferentiation of stromal fibroblasts into myofibroblasts, called "cancer-associated fibroblasts (CAFs)", when stromal fibroblasts preexist in or are recruited into tumor tissue $^{57-59}$. Moreover, TNF- $\alpha$ produced by macrophages has been reported to stimulate the proliferation of fibroblasts in the process of wound healing and acts similarly in tumor tissue to create a fibroblast-rich tumor microenvironment ${ }^{60-62}$. Since the interior region of a tumor rich in fibroblasts is known to contain an abundance of hypoxic regions, a CAF-rich microenvironment is considered to cause radioresistance.

Recent studies, including ours, have revealed the existence of a positive feedback loop by which both CAFs and hypoxic tumor cells enhance the generation of each other $^{63,64}$ and have indicated the importance of this feedback loop in tumor radioresistance. We reported increased expression and secretion of Sonic hedgehog (SHH) protein in pancreatic cancer cells upon exposure to hypoxic stimuli. This increased transcription of $\mathrm{SHH}$ mRNA and secretion of SHH protein were observed to be HIF-1 dependent. SHH protein secreted from pancreatic cancer cells under hypoxia indeed stimulated the Sonic hedgehog signaling pathway in fibroblasts and promoted their growth in a paracrine manner ${ }^{63}$. Such a positive feedback loop may account for the characteristic of pancreatic cancer as the most aggressive malignancy; it is rich in both fibroblasts and hypoxic regions compared with other types of cancer tissues ${ }^{65}$ and is therefore resistant to radiotherapy ${ }^{66}$.

In addition, it has been reported that some factors secreted from CAFs induce radioresistance in cancer cells. For example, CAFs secrete epidermal growth factor (EGF), insulin-like growth factor 2 (IGF2), and fibroblast growth factor 4 (FGF4), which increase not only the proliferation but also the survival of cancer cells after irradiation $^{67}$. It has also been reported that IGF1/2, C-X-C motif chemokine ligand 12 (CXCL12; also called stromal cell-derived factor $1, \mathrm{SDF}-1)$, and $\beta$-hydroxybutyrate secreted from CAFs induce autophagy in irradiated cancer cells and accelerate the recovery and regrowth of tumors after irradiation ${ }^{68}$. In addition, CXCL1 secreted from CAFs was found to cause the accumulation of reactive oxygen species (ROS) in irradiated cancer cells by inhibiting the ROS-scavenging enzyme superoxide dismutase 1 (SOD1), leading to enhanced DNA damage repair in cancer cells ${ }^{69}$. These are just a few examples, and there are other mechanisms responsible for the promotive effects of CAFs on tumor radioresistance.

\section{Discussion}

As summarized here, tumor microenvironments are extremely diverse and significantly different from the microenvironments of normal tissues. For example, not only cancer cells but also a wide variety of other cells are present within malignant solid tumors, forming a unique cellular society. In addition, the oxygen and nutrient environments are not uniform in tumor tissue due to the tumor vascular architecture. Furthermore, there are differences in the tumor microenvironment among cancer types; e.g., kidney cancers are hypervascular ${ }^{70,71}$, and pancreatic cancers are fibrotic/fibroblast-rich ${ }^{66}$. It has become clear in recent years that these environmental factors interact with each other and create a complex intratumoral microenvironment. For the development of novel therapeutic strategies for cancers, it is necessary to understand the nature of the seed and soil hypothesis by identifying the characteristics of tumor microenvironments in a spatiotemporal and global manner and by elucidating the mechanism underlying the induction of malignant phenotypes and therapeutic resistance in cancers.

\begin{abstract}
Acknowledgements
This study was supported by the Funding Program for NEXT Generation WorldLeading Researchers (NEXT Program) from the Japan Society for the Promotion of Science (JSPS), Japan, to H.H. (No. LS071); by the program for Precursory Research for Embryonic Science and Technology (PRESTO) from the Japan Science and Technology Agency (JST) to H.H. (No. JPMJPR14M8); by the Research Project on Development of New Drugs (No. 17933766) and the Translational Research Grant Seeds A (No. A101) to H.H. from the Japan Agency for Medical Research and Development (AMED); by the Core-to-Core Program (JPJSCCA20200009) to H.H. and KAKENHI (Nos. 16H01640, 16H01573, 18KK0241, $19 \mathrm{~K} 22595,2 \mathrm{HO} 03621$, and $20 \mathrm{H} 05033$ to H.H. and $19 \mathrm{KO} 08140$ to J.-M.N.) from the Japan Society for the Promotion of Science (JSPS); and by the research grant programs of the Princess Takamatsu Cancer Research Fund, the Takeda Science Foundation, the Ichiro Kanehara Foundation for the Promotion of Medical Sciences and Medical Care, the Kobayashi Foundation for Cancer Research, and the Foundation for Promotion of Cancer Research to H.H. This study was conducted through the Joint Usage Program of the Radiation Biology Center, Kyoto University, and by that of the Institute for Integrated Radiation and Nuclear Science, Kyoto University. T.S. was a JSPS research fellow, DC2, and was supported by Grants-in-Aid for JSPS Research Fellow (No. 19J14564) from JSPS, Japan.
\end{abstract}

\begin{abstract}
Author details
'Laboratory of Cancer Cell Biology, Graduate School of Biostudies, Kyoto University, Yoshida Konoe-cho, Sakyo-ku, Kyoto 606-8501, Japan. ${ }^{2}$ Department of Genome Repair Dynamics, Radiation Biology Center, Graduate School of Biostudies, Kyoto University, Yoshida Konoe-cho, Sakyo-ku, Kyoto 606-8501, Japan. ${ }^{3}$ Department of Radiation Oncology and Image-applied Therapy, Graduate School of Medicine, Kyoto University, 54 Shogoin Kawahara-cho, Sakyo-ku, Kyoto 606-8507, Japan
\end{abstract}

Conflict of interest

The authors declare no competing interests.

Publisher's note

Springer Nature remains neutral with regard to jurisdictional claims in published maps and institutional affiliations. 
Received: 1 April 2021 Revised: 2 May 2021 Accepted: 3 May 2021. Published online: 16 June 2021

\section{References}

1. Hanahan, D. \& Weinberg, R. A. Hallmarks of cancer: the next generation. Cell 144, 646-674 (2011).

2. Harada, H. How can we overcome tumor hypoxia in radiation therapy? J. Radiat. Res. 52, 545-556 (2011).

3. Yeom, C. J., Goto, Y., Zhu, Y., Hiraoka, M. \& Harada, H. Microenvironments and cellular characteristics in the micro tumor cords of malignant solid tumors. Int. J. Mol. Sci. 13, 13949-13965 (2012).

4. Vaupel, P. Tumor microenvironmental physiology and its implications for radiation oncology. Semin. Radiat. Oncol. 14, 198-206 (2004).

5. Brown, J. M. Evidence for acutely hypoxic cells in mouse tumours, and a possible mechanism of reoxygenation. Br. J. Radio. 52, 650-656 (1979).

6. Dewhirst, M. W. Relationships between cycling hypoxia, HIF-1, angiogenesis and oxidative stress. Radiat. Res. 172, 653-665 (2009).

7. Kimura, H. et al. Fluctuations in red cell flux in tumor microvessels can lead to transient hypoxia and reoxygenation in tumor parenchyma. Cancer Res. $\mathbf{5 6}$ 5522-5528 (1996).

8. Lanzen, J. et al. Direct demonstration of instabilities in oxygen concentrations within the extravascular compartment of an experimental tumor. Cancer Res. 66, 2219-2223 (2006).

9. Vaupel, P., Hockel, M. \& Mayer, A. Detection and characterization of tumor hypoxia using pO2 histography. Antioxid. Redox Signal 9, 1221-1235 (2007).

10. Vaupel, P., Schlenger, K, Knoop, C. \& Hockel, M. Oxygenation of human tumors: evaluation of tissue oxygen distribution in breast cancers by computerized O2 tension measurements. Cancer Res. 51, 3316-3322 (1991).

11. Brown, J. M. \& Wilson, W. R. Exploiting tumour hypoxia in cancer treatment. Nat. Rev. Cancer 4, 437-447 (2004).

12. Kaanders, J. H. et al. Pimonidazole binding and tumor vascularity predict for treatment outcome in head and neck cancer. Cancer Res. 62, 7066-7074 (2002).

13. Gray, L. H., Conger, A. D., Ebert, M., Hornsey, S. \& Scott, O. C. The concentration of oxygen dissolved in tissues at the time of irradiation as a factor in radiotherapy. Br. J. Radiol. 26, 638-648 (1953).

14. Harrison, L. B., Chadha, M., Hill, R. J., Hu, K. \& Shasha, D. Impact of tumor hypoxia and anemia on radiation therapy outcomes. Oncologist 7, 492-508 (2002).

15. Cairns, R. A., Harris, I. S. \& Mak, T. W. Regulation of cancer cell metabolism. Nat. Rev. Cancer 11, 85-95 (2011).

16. Masson, N. \& Ratcliffe, P. J. Hypoxia signaling pathways in cancer metabolism: the importance of co-selecting interconnected physiological pathways. Cancer Metab. 2, 3 (2014).

17. Semenza, G. L. Regulation of cancer cell metabolism by hypoxia-inducible factor 1. Semin. Cancer Biol. 19, 12-16 (2009).

18. Harada, H. Hypoxia-inducible factor 1-mediated characteristic features of cancer cells for tumor radioresistance. J. Radiat. Res. 57(Suppl 1), i99-i105 (2016).

19. Nakashima, R. et al. UCHL1-HIF-1 axis-mediated antioxidant property of cancer cells as a therapeutic target for radiosensitization. Sci. Rep. 7, 6879 (2017).

20. Jiang, P., Du, W. \& Wu, M. Regulation of the pentose phosphate pathway in cancer. Protein Cell 5, 592-602 (2014).

21. Patra, K. C. \& Hay, N. The pentose phosphate pathway and cancer. Trends Biochem. Sci. 39, 347-354 (2014).

22. Meijer, T. W., Kaanders, J. H., Span, P. N. \& Bussink, J. Targeting hypoxia, HIF-1, and tumor glucose metabolism to improve radiotherapy efficacy. Clin. Cancer Res. 18, 5585-5594 (2012).

23. Goto, Y. et al. UCHL1 provides diagnostic and antimetastatic strategies due to its deubiquitinating effect on HIF-1alpha. Nat. Commun. 6, 6153 (2015).

24. Semenza, G. L. Defining the role of hypoxia-inducible factor 1 in cancer biology and therapeutics. Oncogene 29, 625-634 (2010).

25. Aebersold, D. M. et al. Expression of hypoxia-inducible factor-1alpha: a novel predictive and prognostic parameter in the radiotherapy of orophanyngeal cancer. Cancer Res. 61, 2911-2916 (2001).

26. Ishikawa, $\mathrm{H}$. et al. Expression of hypoxic-inducible factor 1alpha predicts metastasis-free survival after radiation therapy alone in stage IIIB cervical squamous cell carcinoma. Int. J. Radiat. Oncol. Biol. Phys. 60, 513-521 (2004).

27. Bindra, R. S., Crosby, M. E. \& Glazer, P. M. Regulation of DNA repair in hypoxic cancer cells. Cancer Metastasis Rev. 26, 249-260 (2007).
28. Olcina, M. M., Grand, R. J. \& Hammond, E. M. ATM activation in hypoxiacauses and consequences. Mol. Cell Oncol. 1, e29903 (2014).

29. Kanu, N. \& Behrens, A. ATMINistrating ATM signalling: regulation of ATM by ATMIN. Cell Cycle 7, 3483-3486 (2008).

30. Uziel, T. et al. Requirement of the MRN complex for ATM activation by DNA damage. EMBO J. 22, 5612-5621 (2003).

31. Bencokova, Z. et al. ATM activation and signaling under hypoxic conditions. Mol. Cell Biol. 29, 526-537 (2009).

32. Hashimoto, T. et al. Severe hypoxia increases expression of ATM and DNAPKcs and it increases their activities through Src and AMPK signaling pathways. Biochem. Biophys. Res. Commun. https://doi.org/10.1016/j. bbrc.2018.09.068 (2018).

33. Barsoum, I. B., Smallwood, C. A., Siemens, D. R. \& Graham, C. H. A mechanism of hypoxia-mediated escape from adaptive immunity in cancer cells. Cancer Res. 74, 665-674 (2014).

34. Zheng, X. et al. Increased vessel perfusion predicts the efficacy of immune checkpoint blockade. J. Clin. Investig. 128, 2104-2115 (2018).

35. Tian, L. et al. Mutual regulation of tumour vessel normalization and immunostimulatory reprogramming. Nature 544, 250-254 (2017).

36. Orimo, A. et al. Stromal fibroblasts present in invasive human breast carcinomas promote tumor growth and angiogenesis through elevated SDF-1/ CXCL12 secretion. Cell 121, 335-348 (2005).

37. Dehne, N., Mora, J., Namgaladze, D., Weigert, A. \& Brune, B. Cancer cell and macrophage cross-talk in the tumor microenvironment. Curr. Opin. Pharm. 35, 12-19 (2017).

38. Brown, J. M., Recht, L. \& Strober, S. The promise of targeting macrophages in cancer therapy. Clin. Cancer Res. 23, 3241-3250 (2017).

39. Sica, A. et al. Macrophage polarization in tumour progression. Semin. Cancer Biol. 18, 349-355 (2008).

40. Cook, P. J. et al. Cox-2-derived PGE2 induces Id1-dependent radiation resistance and self-renewal in experimental glioblastoma. Neuro Oncol. 18, 1379-1389 (2016).

41. Tessner, T. G., Muhale, F., Riehl, T. E., Anant, S. \& Stenson, W. F. Prostaglandin E2 reduces radiation-induced epithelial apoptosis through a mechanism involving AKT activation and bax translocation. J. Clin. Investig. 114, 1676-1685 (2004).

42. Meng, Y. et al. Blockade of tumor necrosis factor alpha signaling in tumorassociated macrophages as a radiosensitizing strategy. Cancer Res. 70, 1534-1543 (2010).

43. Balkwill, F. \& Mantovani, A. Inflammation and cancer: back to Virchow? Lancet 357, 539-545 (2001).

44. Mantovani, A., Sozzani, S., Locati, M., Allavena, P. \& Sica, A. Macrophage polarization: tumor-associated macrophages as a paradigm for polarized M2 mononuclear phagocytes. Trends Immunol. 23, 549-555 (2002).

45. Daurkin, I. et al. Tumor-associated macrophages mediate immunosuppression in the renal cancer microenvironment by activating the 15-lipoxygenase-2 pathway. Cancer Res. 71, 6400-6409 (2011).

46. Gui, Q. L. et al. [lnfiltration of tumor associated macrophages in multiple myeloma and its clinical significance]. Zhonghua Xue Ye Xue Za Zhi 39, 122-127 (2018).

47. Miyake, M. et al. Regulatory T cells and tumor-associated macrophages in the tumor microenvironment in non-muscle invasive bladder cancer treated with intravesical Bacille Calmette-Guerin: a long-term follow-up study of a Japanese cohort. Int. J. Mol. Sci. https://doi.org/10.3390/ijms18102186 (2017).

48. Ooft, M. L. et al. Prognostic role of tumour-associated macrophages and regulatory $T$ cells in EBV-positive and EBV-negative nasopharyngeal carcinoma. J. Clin. Pathol. 71, 267-274 (2018).

49. Matsuoka, Y. et al. The tumour stromal features are associated with resistance to 5-FU-based chemoradiotherapy and a poor prognosis in patients with oral squamous cell carcinoma. APMIS 123, 205-214 (2015).

50. Shi, C. et al. High COX-2 expression contributes to a poor prognosis through the inhibition of chemotherapy-induced senescence in nasopharyngeal carcinoma. Int. J. Oncol. 53, 1138-1148 (2018).

51. Verset, L. et al. Impact of neoadjuvant therapy on cancer-associated fibroblasts in rectal cancer. Radiother. Oncol. 116, 449-454 (2015).

52. Andersen, S. et al. Angiogenic markers show high prognostic impact on survival in marginally operable non-small cell lung cancer patients treated with adjuvant radiotherapy. J. Thorac. Oncol. 4, 463-471 (2009).

53. Mantoni, T. S. et al. Stromal SPARC expression and patient survival after chemoradiation for non-resectable pancreatic adenocarcinoma. Cancer Biol. Ther. 7, 1806-1815 (2008). 
54. Saigusa, S. et al. Cancer-associated fibroblasts correlate with poor prognosis in rectal cancer after chemoradiotherapy. Int. J. Oncol. 38, 655-663 (2011).

55. Coussens, L. M. \& Werb, Z. Inflammation and cancer. Nature 420, 860-867 (2002).

56. Jacobs, T. W., Byrne, C., Colditz, G., Connolly, J. L. \& Schnitt, S. J. Radial scars in benign breast-biopsy specimens and the risk of breast cancer. N. Engl. J. Med. 340, 430-436 (1999).

57. Bhowmick, N. A. et al. TGF-beta signaling in fibroblasts modulates the oncogenic potential of adjacent epithelia. Science 303, 848-851 (2004).

58. Ishii, G. et al. Bone-marrow-derived myofibroblasts contribute to the cancerinduced stromal reaction. Biochem. Biophys. Res. Commun. 309, 232-240 (2003).

59. Singer, A. J. \& Clark, R. A. Cutaneous wound healing. N. Engl. J. Med. 341, 738-746 (1999).

60. Pilling, D., Vakil, V., Cox, N. \& Gomer, R. H. TNF-alpha-stimulated fibroblasts secrete lumican to promote fibrocyte differentiation. Proc. Natl Acad. Sci. USA 112, 11929-11934 (2015).

61. Miyazaki, Y. et al. Expression of a tumor necrosis factor-alpha transgene in murine lung causes lymphocytic and fibrosing alveolitis. A mouse model of progressive pulmonary fibrosis. J. Clin. Investig. 96, 250-259 (1995).

62. Chesney, J., Metz, C., Stavitsky, A. B., Bacher, M. \& Bucala, R. Regulated production of type I collagen and inflammatory cytokines by peripheral blood fibrocytes. J. Immunol. 160, 419-425 (1998).
63. Katagiri, T. et al. HIF-1 maintains a functional relationship between pancreatic cancer cells and stromal fibroblasts by upregulating expression and secretion of Sonic hedgehog. Oncotarget 9, 10525-10535 (2018).

64. Morris, J. P. T., Wang, S. C. \& Hebrok, M. KRAS, Hedgehog, Wnt and the twisted developmental biology of pancreatic ductal adenocarcinoma. Nat. Rev. Cancer 10, 683-695 (2010)

65. Lunardi, S., Muschel, R. J. \& Brunner, T. B. The stromal compartments in pancreatic cancer: are there any therapeutic targets? Cancer Lett. 343, 147-155 (2014).

66. Ryan, D. P., Hong, T. S. \& Bardeesy, N. Pancreatic adenocarcinoma. N. Engl. J. Med. 371, 1039-1049 (2014).

67. Chu, T. Y., Yang, J. T., Huang, T. H. \& Liu, H. W. Crosstalk with cancer-associated fibroblasts increases the growth and radiation survival of cervical cancer cells. Radiat. Res. 181, 540-547 (2014).

68. Wang, Y. et al. Cancer-associated fibroblasts promote irradiated cancer cell recovery through autophagy. EBioMedicine 17, 45-56 (2017).

69. Zhang, $\mathrm{H}$. et al. CAF-secreted CXCL1 conferred radioresistance by regulating DNA damage response in a ROS-dependent manner in esophageal squamous cell carcinoma. Cell Death Dis. 8, e2790 (2017).

70. Choudhary, S., Sudarshan, S., Choyke, P. L. \& Prasad, S. R. Renal cell carcinoma: recent advances in genetics and imaging. Semin. Ultrasound CT MR 30 315-325 (2009).

71. De Meerleer, G. et al. Radiotherapy for renal-cell carcinoma. Lancet Oncol. 15 e170-e177 (2014) 